\title{
Colloidal Nanocrystal Shape and Size Control: the Case of Co
}

\section{Victor F. Puntes ${ }^{1}$, Kannan M. Krishnan ${ }^{2}$, Paul A. Alivisatos ${ }^{1}$}

\begin{abstract}
We show that a relatively simple approach for controlling the colloidal synthesis of anisotropic CdSe semiconductor nanorods can be extended to the size-controlled preparation of magnetic Co nanorods as well as spherically shaped nanocrystals. This approach helps to define a minimum feature set needed to separately control the sizes and shapes of nanocrystals. The resulting Co nanocrystals produce interesting $2 \mathrm{D}$ and $3 \mathrm{D}$ superstructures, including ribbons of nanorods.
\end{abstract}

With the growing interest in building advanced materials using nanoscale building blocks ${ }^{1}$, there is a significant need for general approaches to controlling the sizes and shapes of colloidal inorganic nanocrystals. A range of methods have been employed to this end, but there is still a significant need for general methods that will operate on several chemically distinct systems. Indeed, recently Belcher, $\mathrm{Hu}$, and coworkers ${ }^{2}$ have made the extremely interesting suggestion that combinatorial approaches may have the needed generality. Here we demonstrate that a unified set of relatively simple synthetic strategies can be translated from one nanocrystal system to another. We show that the principles

\footnotetext{
${ }^{1}$ Department of Chemistry, University of California at Berkeley, CA 94720, USA

${ }^{2}$ NCEM, Lawrence Berkeley National Laboratory, CA 94720, USA
} 
that have been developed for independently controlling the size and shape of CdSe nanocrystals ${ }^{3}$ can be directly applied to the technologically important case of cobalt.

We have chosen to study the Co system for several reasons. Co nanocrystals display a wealth of size-dependent structural, magnetic, electronic, and catalytic properties. In particular, the exponential dependence of the magnetization relaxation time on volume has spurred intensive studies of Co nanocrystal synthesis for magnetic storage purposes. It is difficult to make isolated magnetic nanocrystals of $\mathrm{Co}$, in part because the forces between the particles are large. These forces occur both due to the high electron affinity and high surface tension arising from the partially filled d-band, from the large van der Waals forces between polarizable metal particles, and finally from significant magnetic dipole interactions. Nonetheless, there has been considerable progress. Thus, $\mathrm{Co}^{4,5}$ (and the related $\mathrm{CoO}^{6}$ and $\mathrm{FePt}^{7}$ systems) have recently been synthesized by solution phase metal salt reduction. Those particles generally display a multiple twinned crystal structure and chemical contamination from the reducing agent. Metal carbonyl pyrolysis have been largely used for many years to produce $\mathrm{Co}^{8,9}$, $\mathrm{Fe}^{10,11,12}, \mathrm{Ni}^{13}, \mathrm{CoPt}^{14}$ and other magnetic particles, but with a relatively large size distribution. Costly size selective precipitation methods are commonly required in these syntheses in order to obtain narrow enough size dispersion. This prior work suggests that Co is an excellent model system for nanocrystal growth kinetics studies. 
The synthesis of shape controlled CdSe nanocrystals is among the most advanced. For instance, we have recently reported the preparation of a wide range of shapes, including rods, teardrops, tetrapods, and branched tetrapods, all made by relatively simple variations in surfactant composition and time variation of monomer concentration ${ }^{15}$. There are three strategies that we have learned from the prototypical $\mathrm{CdSe}$ system and that we have used here to produce Co nanocrystals with high crystallinity, narrow size distributions, and a high degree of shape control. In both cases the nanocrystals are produced by the injection of an organometallic precursor into a hot surfactant mixture under inert (Ar) atmosphere. The first lesson from $\mathrm{CdSe}$ is that an equilibrium spherical shape will be produced in the presence of a single surfactant under thermodynamic conditions because it minimizes surface area ${ }^{16}$. Second, anisotropic particles, nanorods, are obtained by rapid growth in a surfactant mixture (kinetic conditions), where the different surfactants are used to control the growth rates of different faces selectively ${ }^{3}$. Finally, in both systems it is desirable to separate nucleation from growth via a rapid injection of the precursor and that tight size distributions can be obtained spontaneously when monomers can exchange between the particles, under conditions of "size distribution focusing"16. It is worth noting that Co is a more challenging system because of its richer crystal phase diagram, with three nearly isoenergetic crystal structures (face-centered cubic, hexagonally close packed and epsilon). 
Rapid injection of organometallic reagents in a hot coordinating solvent produces temporally discrete homogeneous nucleation. The decomposition and nucleation occurs rapidly upon injection. The lifetime of monomers in solution is short, and many small metal clusters (nuclei) form simultaneously. The surfactant, by dynamically coating the particles with a close-packed monolayer of coordinating ligand, has the ability to control the size and shape of the growing particles. Through charge transfer, the surface tension is lowered and the growth can be modified. In addition, the surfactant layer prevents the agglomeration of particles, allows monomers to add or subtract, passivates the nanocrystals against oxidation, and defines the minimum interparticle distance.

Consistent with the ideas presented above, rods of hcp-Co are obtained in a binary surfactant mixture at early times after injection (Fig. 1), and these rods spontaneously transform to more thermodynamically stable spheres of $\varepsilon$ - $\mathrm{Co}^{9}$ if they are heated for a sufficient period of time. In these experiments, 0.4 to $0.8 \mathrm{~g}$ of $\mathrm{Co}_{2}(\mathrm{CO})_{8}$, dissolved in $3 \mathrm{ml}$ of o-dichlorobenzene, are injected in a refluxing bath of $12 \mathrm{ml}$ of o-dichlorobenzene $\left(182{ }^{\circ} \mathrm{C}\right)$ in the presence of 0.1 to $0.2 \mathrm{ml}$ of oleic acid and 0.1 to $0.3 \mathrm{~g}$ of trioctylphosphine oxide (TOPO) $)^{17}$. As the reaction proceeds in time, the products evolve in a predictable pattern. Thus, quenching the reaction solution after 5 to $10 \mathrm{~s}$ yields samples with Co nanorods (Fig.1B). In this way we produced macroscopic samples of size-controlled nanoscaled Co nanorods. As time evolves over min, the high-energy hop rod-shaped particles disappear at the expense of monodisperse spherically shaped $\varepsilon$-Co nanocrystals. 
The further from equilibrium at the beginning of the reaction, the longer this process takes. Thus, 30 min were necessary to reach final equilibrium for 4 by $25 \mathrm{~nm}$ rods and up to $60 \mathrm{~min}$ in the case of 4 by $75 \mathrm{~nm}$ rods. Table 1 summarizes the reactants and reaction conditions.

Regarding the changes of the crystal structure, it is well known that for these crystal sizes, temperatures of around $200{ }^{\circ} \mathrm{C}$ are enough to trigger atom diffusion and phase transitions ${ }^{18}$. Even though the hcp structure is the most stable phase for bulk $\mathrm{Co}$ at room temperature, experimental data repeatedly show that $\varepsilon$-Co is the most usually found crystal structure in nanoparticles prepared by wet chemistry $^{4,9}$. The evolution toward a spherical shape is consistent with the high surface tension that reduces the surface to volume ratio. The size of the rods or the spheres appears to be determined by the ratio of surfactant to precursor, consistent with observations in CdSe rod growth as well as in other types of spherical metal nanopartilces ${ }^{19,20}$.

The surfactant mixture of TOPO and oleic acid was used to modulate the relative growth rates of different faces in order to yield rods. At fixed oleic acid concentration, the length of the nanorods is directly proportional to the concentration of TOPO. In the absence of either TOPO or oleic acid, a wide distribution of sizes and shapes has been observed. TOPO is also required to promote atom exchange between particles, a necessary feature for size distribution focusing and kinetic control - nanocrystals of Co fully decompose to monomeric species upon refluxing in TOPO. 
All of the rods investigated by high resolution transmission electron microscopy (HRTEM) showed that the long direction is parallel to the (101) planes. Thus, the TOPO must selectively stabilize the (101) face of Co, effectively decreasing its relative growth rate. These observations are supported by X-ray powder diffraction (XRD) studies (Fig.1A), where the 101 reflection shows a smaller intensity than expected and a larger width than the other reflections. However, the TEM data appears to be more definitive in this case, because similar XRD patterns could be obtained by the superposition of hep and $\varepsilon$-Co patterns.

In the equilibrium samples, the spherical, single crystalline Co particles, display a complex cubic primitive structure, recently discovered ${ }^{4,9}$ called $\varepsilon$-Co ${ }^{21}$. The full width at medium height of the XRD peaks of Fig. $1 \mathrm{~F}$ indicated an average crystal size of $73 \AA$, which roughly corresponds to the particle size $(8 \mathrm{~nm})$ observed in the TEM pictures (Fig.1E). HRTEM and dark-field TEM also showed that the particles are defect-free single crystals ${ }^{22}$.

Proof of the quality of the samples yielded by this approach comes from observations of the spontaneous self-assembly of the nanocrystals, as well as from the formation of unique superstructures, such as ribbons of rods. All of the sizes and shapes self-assemble into superstructures as a colloidal solution is allowed to evaporate slowly in a controlled atmosphere. A drop of the colloids ( $2 \%$ weight of particles in the volume) was put onto a carbon-coated TEM grid at room temperature and slowly evaporated (in a covered watchglass). The use of high boiling solvents (like o-dichlorobenzene), allows slow evaporation at RT, which permits the particles to diffuse to their lowest energy sites during 
evaporation, producing well-defined super-structures (Figs.2 and 3). The final arrangement of the nanocrystal assembly is driven by the balance of surface tension, van der Waals forces, and magnetic interactions among superparamagnetic (SP) or ferromagnetic (FM) particles.

Spherical particles self-assemble into hexagonal 2D superlattices (Fig.2A) ${ }^{4,5,20}$. This assembly is determined by the size of the crystals and the thickness of the coating layer $(\sim 2 \mathrm{~nm})$. Higher initial concentrations lead to 3D self-assemblies where the particles in the second layer occupy sites determined by the hexagonal arrangements (Fig.2B). Small magnetic particles are single domain, i.e., all the atomic magnetic spins of the particle are coupled in the same direction and the particle behaves as a single magnetic dipole. Depending on anisotropy, size and temperature, the magnetic dipole of the particles will be free to rotate (superparamagnetism) or will be blocked in the anisotropy direction (ferromagnetism). In the first case, the time average of the magnetic moment of the particle is zero. The relation between anisotropy energy and thermal energy $\left(\mathrm{KV}=\mathrm{k}_{\mathrm{B}} \mathrm{T}\right)^{23}$, determines the transition between the superparamagnetic (SP) and the ferromagnetic (FM) regime ${ }^{24}$. Once the particles become FM, magnetic interactions among them start to play a crucial role following the relation between magnetic interaction and thermal energy $\left(2 \mu^{2} / a^{3}=k_{B} T\right)^{25}$. Thus, due to strong dipole-dipole interactions, the nanocrystals form closed loops in order to minimize the magnetostatic energy (Fig2c-f). In the transition range, a mixture of hexagonal monolayer and closed loops is observed (Fig.2c-d) suggesting that in this case, the SP-FM transition is taking place at room temperature for particles 
with sizes around $12 \mathrm{~nm}$,. For larger particles, $16 \mathrm{~nm}$ in diameter (Fig.2e-f), nose-to-tail arrangements of the individual particles appear covering the whole sample, owing to their larger magnetic moment ${ }^{8,26}$. The magnetic forces thus place strong limits on the types of self-assembled structures which can be arisen.

A different type of superstructure is observed in the case of magnetic nanorods, namely long ribbons of rods (Fig.3). In this case, the constituent nanorods are large enough to be ferromagnetic, while they are still single domain ${ }^{24,27}$. Furthermore, a strong shape anisotropy due to its rod shape favors the blocking of the magnetic moment in the long direction of the particle at room temperature ${ }^{24}$. In these assemblies, the magnetic dipoles should be in an alternating up and down arrangement in order to minimize magnetostatic energy ${ }^{27}$. The length of these ribbons (typically of about $300 \mathrm{~nm}(60$ rods)) is determined by the magnetic properties of the single rods. Magnetic imaging and magnetic characterization are being carried out now to elucidate these points. Regarding magnetic storage technology, it has been suggested that nanorod assemblies would be suitable for reaching the one-bit-per particle $\operatorname{limit}^{28}$. The antiferromagnetic coupling we observe between the rods may place constraints on this.

We can now describe a minimum set of requirements to achieve size and shape control of inorganic nanocrystals, in general. There must be a suitable organometallic precursor that rapidly decomposes to yield monomers at 
temperatures where the surfactants are stable. Two surfactants must be found, which differentially adsorb to the nanocrystal faces, allowing for rod formation. One of the surfactants must promote monomer exchange between particles, to allow for size distribution focusing. 


\section{REFERENCES}

1. C. M. Lieber, Sloid State Commun. 107, 607 (1998).

2. S. R. Whaley, D. S. English, E. L. Hu, P. F. Barbara and A. M. Belcher, Nature 405, $665(2000)$

3. X. Peng et al., Nature 404, 59 (2000).

4. S. Sun, C. B. Murray, J. Appl. Phys. 85, 4325 (1999).

5. C. Petit, A.Taleb, P. Pileni, J. Phys. Chem. 103, 1805 (1999).

6. J. S. Yin, Z.L. Wang, Phys. Rev. Lett. 79, 2570 (1997).

7. S. Sun, C. B. Murray, D. Weller, L. Folks, A. Moser, Science 287, 1989 (2000).

8. J. R. Thomas, J. Appl. Phys. 37, 2914 (1966).

9. D. P. Dinega, M. G. Bawendi, Angew. Chem. Int. ed. 38, 1788 (1999)

10. J. R. Thomas, US Patent no. 3284358, 1966/11/08.

11. D. de Caro et al., Chem. Mater. 8, 1987 (1996)

12. S. J. Park et al., J. Am. Chem. Soc. 122, 8581 (2000).

13. T. Ould Ely, C. Amiens, B. Chaudret, Chem. Mater. 11, 526 (1999)

14. T. Ould Ely et al., J. Phys. Chem. B 104, 165 (2000)

15. L. Manna, E. C. Scher, A. P. Alivisatos, J. Am. Chem. Soc. (in press. Available on line).

16. X. Peng, J. Wickham, A. P. Alivisatos, J. Am. Chem. Soc. 120, 5343 (1998).

17. This synthesis was carried out using standard airless procedures and commercially available reagents. $\mathrm{Co}_{2}(\mathrm{CO})_{8}$ stabilized in hexane, oleic acid 
(99\%), o-Dichlorobenzene (98\%) were purchased from Aldrich. TOPO (90\%) was purchased from Alfa Aesar.

18. M. H. Yang, C. P. Flynn, Phys. Rev. Lett. 62, 1476 (1989).

19. G. Schmid, F. L. Chi, Adv. Mater 10, 515 (1998)

20. Z. L. Wang, Adv. Mater 10, 13 (1998).

21. $\varepsilon$-Co , consist of a complex cubic primitive structure $\left(P 4_{1} 32, a=6.09 \AA\right)$, isotypic related to the beta phase of manganese, with 20 atoms present in the elemental cell.

22. It is well know that in hep Co the sliding of atomic planes is a low energy process and often causes Co crystals to display stacking faults. In the case of $\varepsilon$ Co, it appears that there are no stacking faults, probably due to the complicated crystal structure where sliding a crystal plane is energetically more costly. X-ray emission spectroscopy (EDAX) and electron energy-loss spectroscopy (EELS) using nanometer-scale electron probes in a TEM confirmed that the particles are pure $\mathrm{Co}$ and no evidence for the formation of $\mathrm{CoO}$ or $\mathrm{Co}_{3} \mathrm{O}_{4}$ has been observed.

23. Where $\mathrm{K}$ is the anisotropy constant, $\mathrm{V}$ the particle volume, $\mathrm{k}_{\mathrm{B}}$ the boltzman constant and $\mathrm{T}$ the temperature.

24. J. L. Dorman, D. Fiorani, E. Tronc, Adv. in Chem. Phys. 98, 283 (1997).

25. Where $\mu$ is the magnetic dipole moment and $\mathrm{a}$ is the particle diameter, $\mathrm{k}_{\mathrm{B}}$ the boltzman constant and $\mathrm{T}$ the temperature.

26. for a detailed study of the magnetic properties of those particles, see: V. Puntes, P. Alivisatos, K. Krishnan, Appl. Phys. Lett. (in press); V. Puntes and K. Kannan, IEEE Trans. Magn. (in press) 
27. S. Hamzaoui, M. Labrune, I. B. Puchalska, J. Magn. Magn. Mat. 22, 69 (1980).

28. F. J. Himpsel, J. E. Ortega, G. J. Mankey, R. F. Willis, Adv. in Phys. 47, 511 (1998).

29. This work was supported by the NIH National Center for Research Resources, Grant Number 1 R01 RR-14891-01 through the U.S. Department of Energy under Contract No. DE-AC03-76SF00098. 


\section{FIGURE CAPTIONS}

Fig.1. Evolution of crystal structure, size and shape with time after the injection of a solution of $0.6 \mathrm{~g}$ of $\mathrm{Co}_{2}(\mathrm{CO})_{8}$ in $3 \mathrm{ml}$ of o-dichlorobenzene into $0.1 \mathrm{ml}$ oleic acid and 0.2 g TOPO in $12 \mathrm{ml}$ o-dichlorobenzene, at $182{ }^{\circ} \mathrm{C}$ : (A) XRD pattern at $15 \mathrm{~s}$ corresponding to a hep Co crystal structure (B) TEM microgaph of the solution at $15 \mathrm{~s}$ (C) at $100 \mathrm{~s}$ (D) at $300 \mathrm{~s}$ and (E) at $1800 \mathrm{~s}$. (F) XRD pattern at $1800 \mathrm{~s}$ corresponding to a $\varepsilon$-Co crystal structure. Bar, $100 \mathrm{~nm}$.

Fig.2 (A) Monolayer and (B) bilayer of $10 \mathrm{~nm} \varepsilon$-Co nanoparticles. Reactants: $0.45 \mathrm{~g}$ of $\mathrm{Co}_{2}(\mathrm{CO})_{8}$ in $3 \mathrm{ml}$ of o-Dichlorobenzene and $0.2 \mathrm{ml}$ Oleic Acid and $0.1 \mathrm{~g}$ of pure TOPO in $12 \mathrm{ml}$ o-Dichlorobenzene. (C) and (D) $12 \mathrm{~nm} \varepsilon$-Co particles. Reactants: $0.54 \mathrm{~g}$ of $\mathrm{Co}_{2}(\mathrm{CO})_{8}$ in $3 \mathrm{ml}$ of o-Dichlorobenzene and $0.2 \mathrm{~m}$. Oleic Acid and $0.1 \mathrm{~g}$ of pure TOPO in $12 \mathrm{ml}$ o-Dichlorobenzene. (E) and (F) $16 \mathrm{~nm}$ e-Co particles. Reactants: $0.65 \mathrm{~g}$ of $\mathrm{Co}_{2}(\mathrm{CO})_{8}$ in $3 \mathrm{ml}$ of o-Dichlorobenzene and $0.2 \mathrm{~m}$. Oleic Acid and $0.1 \mathrm{~g}$ of pure TOPO in $12 \mathrm{ml}$ o-Dichlorobenzene. Bar, $100 \mathrm{~nm}$.

Fig.3. (A-C) Assemblies of (in average) 4 x $25 \mathrm{~nm}$ hcp Co rods. Reactants: $0.6 \mathrm{~g}$ of $\mathrm{Co}_{2}(\mathrm{CO})_{8}$ in $3 \mathrm{ml}$ of o-Dichlorobenzene and $0.1 \mathrm{ml}$ Oleic Acid and $0.2 \mathrm{~g}$ of technical TOPO in $12 \mathrm{ml}$ o-Dichlorobenzene. (D-F) Assemblies of (in average) 4 x $75 \mathrm{~nm} \mathrm{hcp} \mathrm{Co}$ rods. Reactants: $0.6 \mathrm{~g}$ of $\mathrm{Co}_{2}(\mathrm{CO})_{8}$ in $3 \mathrm{ml}$ of o-Dichlorobenzene and $0.1 \mathrm{ml}$ Oleic Acid and $0.3 \mathrm{~g}$ of technical TOPO in $12 \mathrm{ml}$ o-Dichlorobenzene. Bar, $100 \mathrm{~nm}$. 
